\title{
The Effect of Current Inhomogeneity on the Performance and Degradation of Li-S Batteries
}

\author{
I. Hunt, ${ }^{\text {a } T . ~ Z h a n g, ~}{ }^{\text {a }}$ Y. Patel, ${ }^{a}$ M. Marinescu, ${ }^{\text {a }}$ R. Purkayastha, ${ }^{\mathrm{b}}$ P. Kovacik, ${ }^{\mathrm{b}}$ S. Walus, ${ }^{\mathrm{b}}$ \\ A. Swiatek, ${ }^{\text {b }}$ and G. J. Offer $\mathbb{D}^{\text {a,z }}$ \\ ${ }^{a}$ Department of Mechanical Engineering, Imperial College London, SW7 2AZ, United Kingdom \\ ${ }^{b}$ OXIS Energy Ltd, E1 Culham Science Centre, Abingdon, OX14 3DB, United Kingdom
}

\begin{abstract}
The effect of thermal gradients on the performance and cycle life of Li-S batteries is studied using bespoke single-layer Li-S cells, with isothermal boundary conditions maintained by Peltier elements. A temperature difference is shown to cause significant current imbalance between parallel connected single-layer cells, causing the hotter cell to provide more charge and discharge capacities during cycling. During charge, significant shuttle is induced in the hotter Li-S cell, causing accelerated degradation of it. A bespoke multi-tab cell in which the inner layers are electrically connected to different tabs versus the outer layers, is used to demonstrate that noticeable current inhomogeneity occurs during the operation of practical multilayer Li-S pouch cells, which is expected to affect their performance and degradation. The observed thermal and current inhomogeneity should have a direct consequence on battery pack and thermal management system design for real world Li-S battery packs.

(C) The Author(s) 2017. Published by ECS. This is an open access article distributed under the terms of the Creative Commons Attribution 4.0 License (CC BY, http://creativecommons.org/licenses/by/4.0/), which permits unrestricted reuse of the work in any medium, provided the original work is properly cited. [DOI: $10.1149 / 2.0141801$ jes] All rights reserved.

(cc) BY
\end{abstract}

Manuscript submitted August 21, 2017; revised manuscript received November 13, 2017. Published November 29, 2017. This paper is part of the JES Focus Issue on Lithium-Sulfur Batteries: Materials, Mechanisms, Modeling, and Applications.

Lithium-sulfur (Li-S) batteries are a promising post lithium-ion battery technology due to their high theoretical energy density of 2600 $\mathrm{Wh} / \mathrm{kg}$ along with other potential benefits such as low cost, improved safety and good low-temperature performance. ${ }^{1-4}$ The main challenges in the commercialization of Li-S batteries are to improve their cycle life, rate performance, charging efficiency, and high-temperature performance. ${ }^{5,6}$

The performance of Li-S batteries depends on a number of temperature-dependent mechanisms. During discharge, lithium and sulfur react electrochemically to form soluble polysulfide species of different chain lengths, such as $\mathrm{Li}_{2} \mathrm{~S}_{6}$ and $\mathrm{Li}_{2} \mathrm{~S}_{4}$, which are then reduced further to produce $\mathrm{Li}_{2} \mathrm{~S}$, which is insoluble and precipitates. When the cell is subsequently charged, the precipitated $\mathrm{Li}_{2} \mathrm{~S}$ must re-dissolve before being oxidized back to sulfur and lithium. The solubility of polysulfides as well as the rates of precipitation and dissolution are dependent on temperature: ${ }^{7,8}$ high temperatures promote dissolution of both sulfur and $\mathrm{Li}_{2} \mathrm{~S}$, whereas low temperatures limit dissolution and encourage precipitation. Model predictions indicate that a decrease in the solubility of polysulfides and their dissolution rates leads to reduced discharge and charge capacities for $\mathrm{Li}-\mathrm{S}$ batteries. ${ }^{9}$ The power capability of Li-S batteries is also critically dependent on temperature. At low temperatures, $\mathrm{Li}-\mathrm{S}$ cells suffer from larger polarization from reduced ionic conductivity and diffusion rates, resulting in lower rate performance. ${ }^{10}$

The polysulfide shuttle, a characteristic phenomenon of the Li-S battery, is itself temperature-dependent. The shuttle is strongly correlated to many of the performance challenges that inhibit the mainstream uptake of Li-S batteries, ${ }^{5}$ such as the self-discharge, low charge efficiency and irreversible degradation of Li-S batteries at high statesof-charge (SoC). Shuttle occurs when the highly soluble dissolved long chain polysulfides migrate to the lithium anode, where they react to form shorter chain polysulfides; these diffuse back to the cathode, where they are oxidized again during charge. The shuttle reaction corrodes the metallic lithium anode, where it causes irreversible deposition of short-chain polysulfides, leading to irreversible capacity loss. ${ }^{11}$ The rate of shuttle was shown to increase with temperature due to the increase in the solubility and diffusion rates of long-chain polysulfides. ${ }^{12}$ As a result, while Li-S batteries exhibit higher en-

${ }^{\text {zE} E-m a i l: ~ g r e g o r y . o f f e r @ i m p e r i a l . a c . u k ~}$ ergy and power at high temperatures, they also suffer from faster self-discharge, lower Columbic efficiency, and faster degradation.

Like other battery chemistries, Li-S batteries generate three types of heat: resistive, entropic, and reaction heat. It was found via isothermal calorimetry that the heat generation rate during cell discharge follows the same trend as its resistance variation, ${ }^{13}$ indicating the irreversible resistive heat as the dominant heat generation mechanism. During slow-rate charge the major heating mechanism was found to be caused by shuttle. ${ }^{12}$ At high temperatures and low charge rates, the rate of shuttle can become comparable or even larger than the rate of charge, leading to a flat charge voltage and a seemingly infinite charge capacity. In the shuttle-dominated charging region, the internal shuttle current can generate more heat than the cell dissipates, leading to an increase in cell temperature and potentially to thermal runaway. The safety of $\mathrm{Li}-\mathrm{S}$ batteries at high temperatures is of particular concern due to the low boiling and flashing points of the commonly used solvents. ${ }^{8}$

Cell heating is expected to increase in Li-S battery packs in applications, due to thermal boundary conditions and practical limits in the number of cells monitored. It is thus important to thermally manage $\mathrm{Li}-\mathrm{S}$ cells in real-world applications in order to reach a compromise between power, cycle life, and safety. Cells in a battery pack are typically cooled via the cell surface or the cell tabs with forced air convection or liquid coolant. Such thermal management unavoidably produces temperature gradients within the battery pack, as well as within each cell, which can influence both performance and degradation. ${ }^{14}$ For example, surface cooling causes the inner layers of the cell to be hotter than the outer ones, as shown for lithium ion batteries by measurements from thermocouples inserted in a battery and predictions of thermally-coupled electrochemical battery models. ${ }^{15}$ Such thermal gradients have a negative effect on the cell cycle life. The hotter inner cell layers discharge and age faster than the cooler outer layers, leading to a positive feedback for thermal and electrical inhomogeneity among the cell layers that accelerates the aging of the battery. As a result, for conventional lithium-ion cells, surface cooling can lead to faster degradation than tab cooling at high cycling rates. ${ }^{14}$

The effect of thermal gradients and the induced current inhomogeneity have not been explored for Li-S cells, but they are expected to affect battery behavior in real applications, where temperature difference is present across a battery pack as well as among the layers of a single cell. Here the effect of thermal gradients on the performance and cycle life of Li-S batteries is studied using bespoke single-layer 


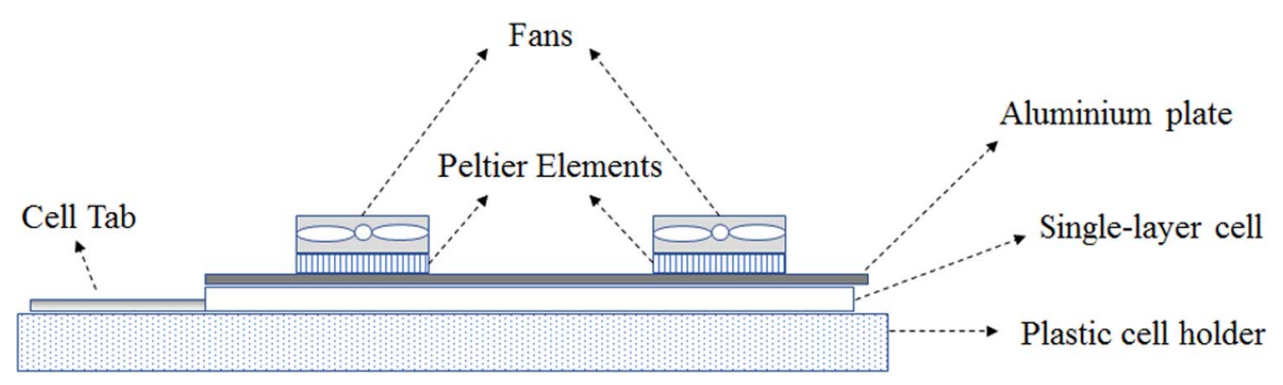

Figure 1. Schematic of the temperature control rig. Peltier elements are used to control the temperature on one surface of the single layer pouch cell.

Li-S cells, with iso-thermal boundary conditions maintained by watercooled Peltier elements. The unique resistance profile and the shuttle behavior of Li-S cells is shown to cause a strong positive feedback for current inhomogeneity among cells held at different temperatures, causing accelerated degradation of the hotter cells. With a tailor-made multilayer Li-S cell whose inner layers and outer layer are connected to separate tabs, it is demonstrated that noticeable current inhomogeneity can occur during the operation of practical Li-S pouch cells, which is expected to affect their performance and degradation. Our results highlight the importance of maintaining a uniform temperature across Li-S cells and battery packs, requiring an effective thermal management system.

\section{Experimental}

Single-layer cells.-In order to investigate the performance of Li-S batteries under isothermal conditions, pouch cells with a single anode-cathode pair were manufactured by Oxis Energy Ltd. The cells are $0.41 \mathrm{~mm}$ thick (measured using a Moore \&Wright MW201-01DAB outside micrometer), and have a nominal capacity of $0.211 \mathrm{Ah}$. The cells contain a sulfone-based solvent and the electrolyte/sulfur ratio has been optimized in order to deliver maximum cell-level specific energy density.

The cells were placed in the temperature control rig described below, at $20^{\circ} \mathrm{C}, 30^{\circ} \mathrm{C}$ and $40^{\circ} \mathrm{C}$, and cycled at $0.1 \mathrm{C}(0.633 \mathrm{~A})$ charge and $0.2 \mathrm{C}(1.266 \mathrm{~A})$ discharge between $1.5 \mathrm{~V}$ and $2.45 \mathrm{~V}$ with an 11 hour time cutoff for charging using a Biologic BCS-815 battery cycler. A current pulse of $10 \mathrm{~s}$ duration and $10 \%$ amplitude on top of the constant charge/discharge current was introduced every $2 \%$ SoC (measured by charge throughput) during charge/discharge. The resulting change in voltage was measured $10 \mathrm{~ms}$ after the change in current was applied and was used to calculate the internal resistance. The extra current was considered negligible.

Temperature control rig.-A schematic of the temperature control rig is shown in Fig. 1. Peltier elements were used to control the temperature of the single layer cells. The Peltier elements were powered by bi-directional motor controllers (SyRen10) and the temperature was measured directly underneath each Peltier element using thermocouples connected to a PICO TC-08 logger. The surface temperature was controlled via a custom-made C\# computer program employing PID control of the motor controllers powering the Peltier elements. Before testing, additional thermocouples were placed on the cell to ensure that the temperature is homogeneous across the surface. A maximum difference of $0.6^{\circ} \mathrm{C}$ was allowed. The cells were thin enough that no appreciable temperature difference through the cell was observed.

Parallel-connected cells.-In order to monitor the difference between the state of cells that are connected in parallel but are at different temperatures, a test rig was designed to hold three single layer cells connected via small shunt resistors; these resistors allow one to measure the current provided by each cell. The resistances of the external current paths were carefully balanced to ensure they didn't cause any current inhomogeneity. A schematic of this set-up is shown in Fig. 2a. The temperature of each cell was controlled via two Peltier elements, as in Fig. 1. The voltage across the shunt resistors (Arcol HS25 R02$20 \mathrm{~m} \Omega$ ) was measured using a PICO ADC-24, and used to calculate the current through each cell.

The parallel connection of single layer cells was cycled between $1.5 \mathrm{~V}$ and $2.45 \mathrm{~V}$ with a $0.1 \mathrm{C}$ charge current and a $0.2 \mathrm{C}$ discharge current, while holding the three cells at different temperatures (20,

(a)

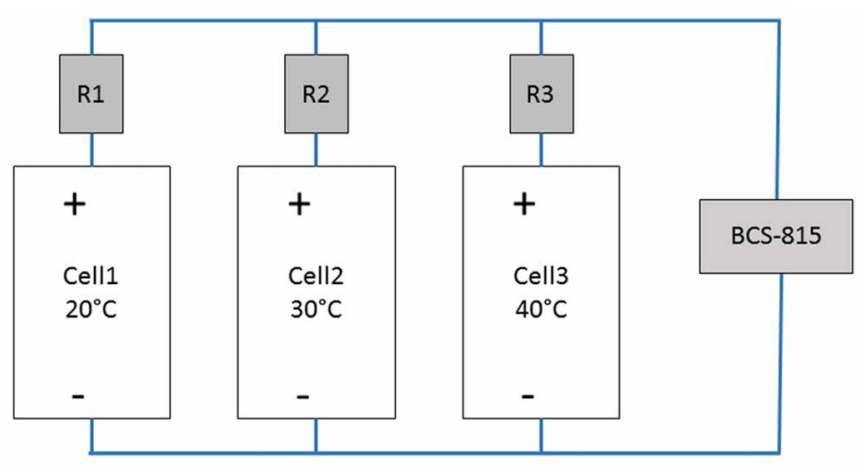

(b)

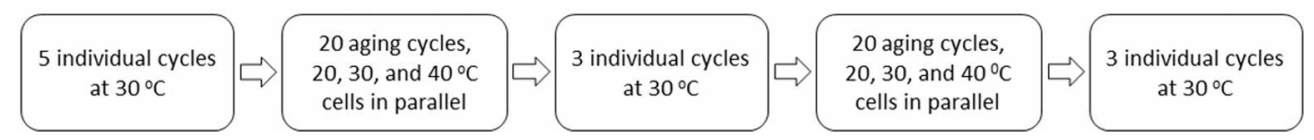

Figure 2. (a) Schematic of single layer cells connected in parallel and held at different temperatures. The current through each cell is calculated from the voltage drop across each of the three shunt resistors $\mathrm{R}_{1}, \mathrm{R}_{2}, \mathrm{R}_{3}$. (b) The experimental sequence for individual characterization cycles at $30^{\circ} \mathrm{C}$ and parallel connection aging cycles at different temperatures. 


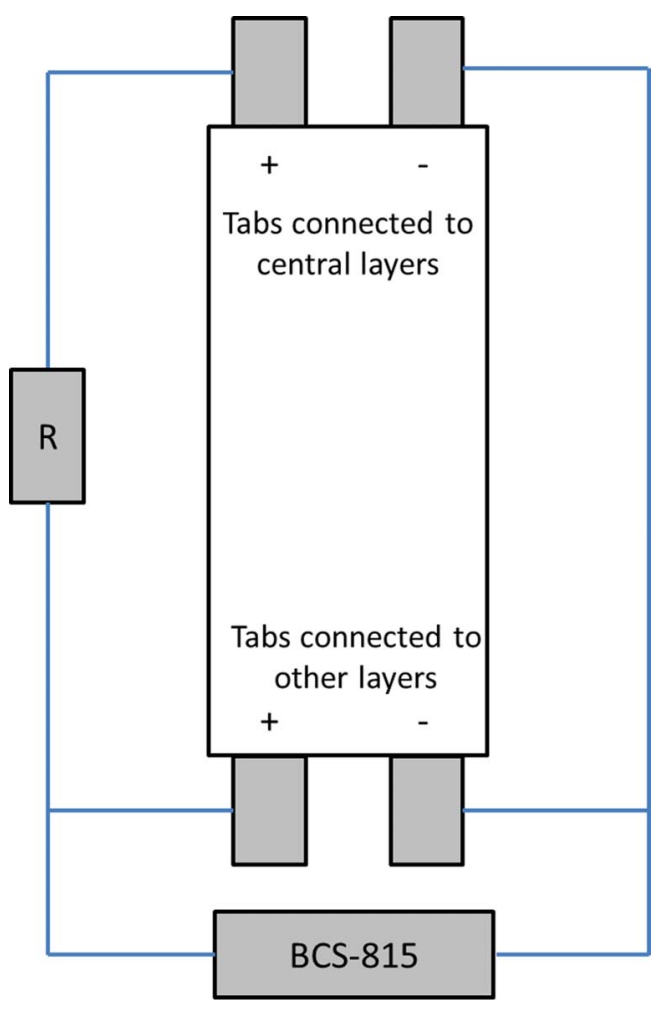

Figure 3. Schematic of the bespoke multilayer cell set-up. A pair of tabs is connected only to the inner three layers of the cell, while the other is connected to the other 25 layers. The battery cycler is connected to all layers, while the current going through the middle three layers is calculated from the voltage drop measured across the shunt resistor $\mathrm{R}$.

30 , and $40^{\circ} \mathrm{C}$ ). After 20 cycles the cells were disconnected and cycled individually at $30^{\circ} \mathrm{C}$, in order to assess their level of degradation. The cells were then connected in parallel and cycled at their respective different temperatures again for another 20 cycles, before being cycled individually again at $30^{\circ} \mathrm{C}$ (Fig. 2b).

Multi-tab cell.-In order to verify the presence of current inhomogeneity in an application-relevant system, a bespoke multiple layer pouch cell was manufactured. The central three layers were connected to a second pair of tabs, separate from the pair of tabs connected to the other layers of the cell. The cell had 28 layers in total and a nominal capacity of $6.402 \mathrm{Ah}$. The current flow to the central layers of the cell was measured using a small shunt resistor, as shown schematically in Fig. 3. During charge and discharge, the cell was placed between two cooling plates which were held at $30^{\circ} \mathrm{C}$ using Peltier elements, as in Fig. 1. The cell was pre-cycled 4 times at $30^{\circ} \mathrm{C}$ using a $0.2 \mathrm{C}$ discharge current and a $0.1 \mathrm{C}$ charge current between $1.5 \mathrm{~V}$ and $2.45 \mathrm{~V}$.

\section{Results and Discussion}

Single-layer cells. - The single layer cells are thin and have a small enough thermal mass to ensure that the temperature gradient within each cell is negligible under the temperature control by Peltier elements. Fig. 4 shows the voltage curves during charge and discharge of a single layer cell held at different temperatures, and the corresponding ohmic resistance. All discharge curves exhibit an upper and a lower voltage plateau. However, the shape of the lower plateau at $20^{\circ} \mathrm{C}$ is significantly different from the typical flat voltage profile, retrieved at $30^{\circ} \mathrm{C}$ and $40^{\circ} \mathrm{C}$. At $20^{\circ} \mathrm{C}$ the voltage continues to decrease beyond the expected end of upper plateau, while the lower plateau appears as a hump due to a noticeable voltage rise. This hump-shaped lower discharge plateau has been reported for Li-S cells with cathodes prepared by the phase inversion method and with a relatively low elec- a)
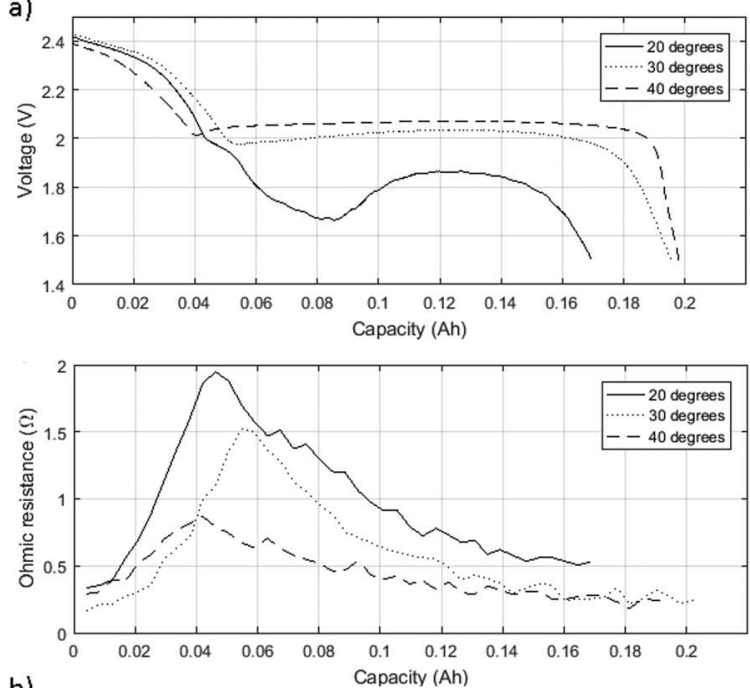

b)
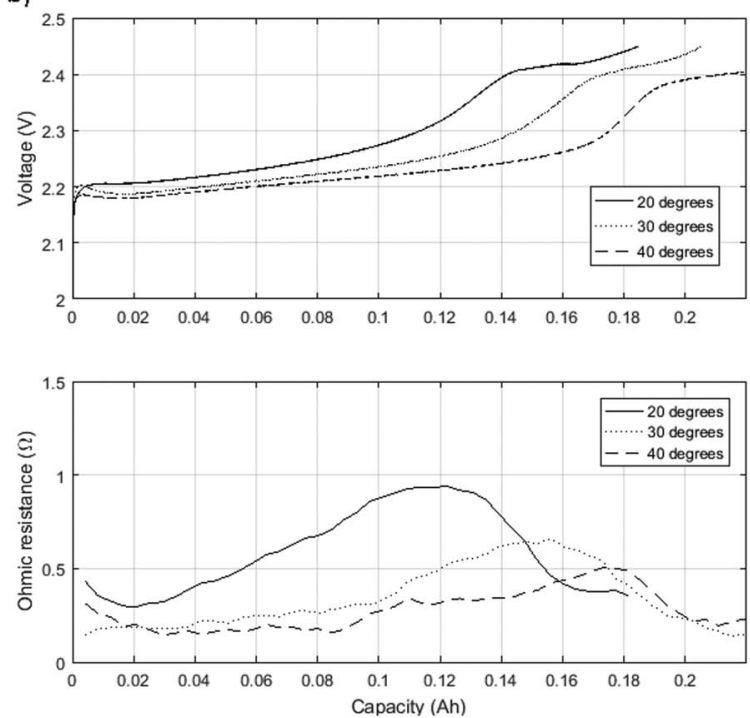

Figure 4. Voltage curves and resistances of $0.211 \mathrm{Ah}$ single layer cells held at various temperatures during (a) a $0.2 \mathrm{C}$ discharge, and (b) a $0.1 \mathrm{C}$ charge. The cell temperature has a significant effect on its ohmic resistance and capacity.

trolyte uptake. ${ }^{16,17}$ It was hypothesized that the hump was caused by increased $\mathrm{Li}^{+}$transport overpotential due to the limited accessibility of electrolyte into the porous cathode. The increased transport overpotential should also occur here at the lower temperature of $20^{\circ} \mathrm{C}$ due to reduced ionic diffusivity and electrolyte conductivity. However, the discharge hump was not observed in other studies using pouch cells at low temperatures ${ }^{10,18,19}$. It is possible that such behavior is obscured in larger, multilayer Li-S cells, due to the existence of a temperature gradient and associated differences in resistance and state of charge of the cell layers.

Under the three different temperatures, the upper plateau of the discharge voltage has a similar shape but accounts for considerably different capacities. At $40^{\circ} \mathrm{C}$ the upper-plateau has the smallest capacity, probably caused by an increased shuttle rate at this relatively high temperature, which leads to self-discharge of the cell during the upper plateau. Despite having the smallest upper-plateau capacity, the $40^{\circ} \mathrm{C}$ cell has the largest lower-plateau capacity as a result of reduced polarization from charge transfer and ionic transport at elevated temperatures. At $20^{\circ} \mathrm{C}$, the upper plateau capacity is again smaller than that at $30^{\circ} \mathrm{C}$, likely due to increased total cell resistance at the lower temperature.

The ohmic resistance of the cell during discharge, shown in Fig. $4 \mathrm{a}$, generally decreases with increasing temperature. The resistance 
a)

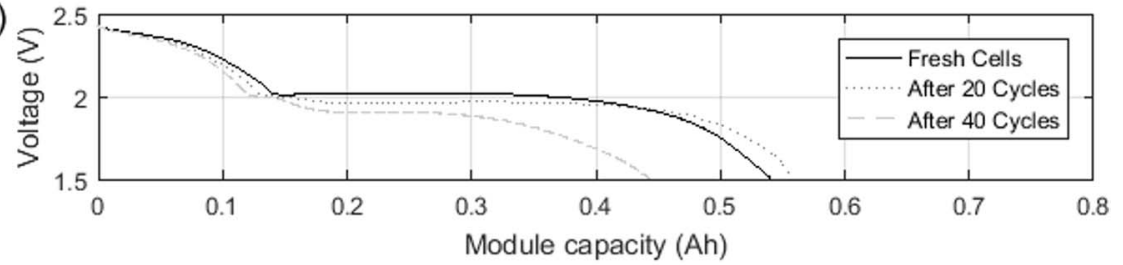

b)

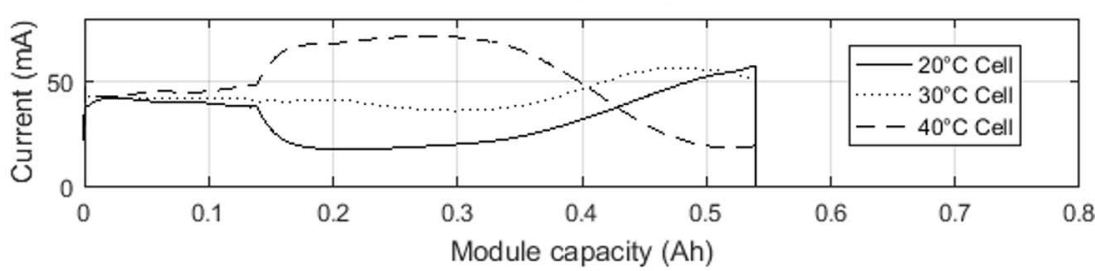

c)

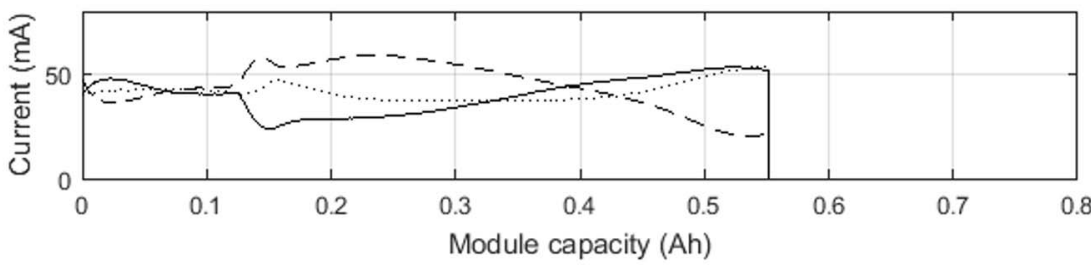

d)

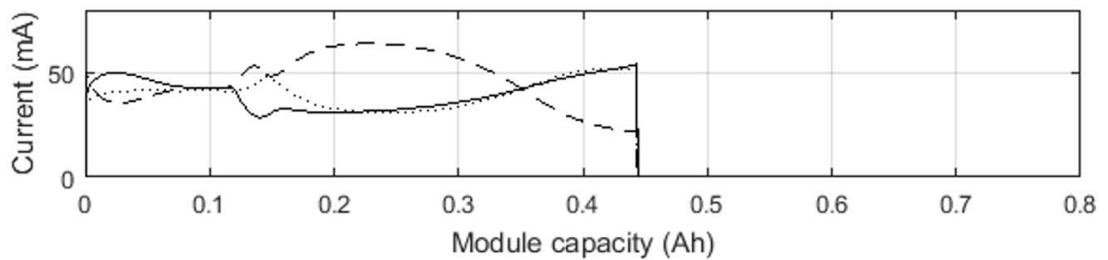

Figure 5. (a) Discharge voltage of three parallelconnected $0.211 \mathrm{Ah}$ single layer cells, and individual current through each of them during (b) cycle 1 , (c) after 20 ageing cycles, and (d) after 40 aging cycles for an $0.2 \mathrm{C}$ discharge current with $1.5 / 2.45 \mathrm{~V}$ voltage cutoff. was calculated $10 \mathrm{~ms}$ after a current change hence it is assumed to be dominated by ohmic resistance and not to include significant contributions from charge transfer and diffusion resistances. The charge transfer and diffusion resistances increase throughout the discharge and become dominating toward the end of discharge, leading to large overpotentials and thus causing the end of discharge as a result of the voltage cut-off being reached. ${ }^{20}$ As expected, a peak in the resistance appears at all temperatures at the transition between upper and lower plateaus, associated with a maximum in electrolyte resistance, as a result of high concentrations of dissolved polysulfides in this state. ${ }^{21,22}$ The size and location of this peak is strongly temperature dependent. In the case of discharge at $20^{\circ} \mathrm{C}$, the maximum in resistance does not correspond to the minimum in voltage, but to the expected location of the transition between plateaus.

As shown in Fig. 4b, the charging voltage is similar for the three temperatures, with the $20^{\circ} \mathrm{C}$ charge exhibiting the smallest apparent charge capacity and the $40^{\circ} \mathrm{C}$ showing the largest. This larger apparent charge capacity is at least partly due to an increased effect of shuttle, supported by the observation that at $40^{\circ} \mathrm{C}$ the voltage cutoff is not reached within the $11 \mathrm{hrs}$ time limit. Also during charge, the ohmic resistance peaks at the transition between the lower and upper plateaus, as shown in Fig. 4b. The location of the ohmic resistance peak is different because shuttle rate and cell resistance are temperature-dependent.

The measurements on single-layer Li-S cells show performance that is strongly temperature-dependent, with voltage, resistance and capacity changing significantly between $20^{\circ} \mathrm{C}$ and $40^{\circ} \mathrm{C}$. The transitions between the upper and lower plateaus and thus the locations of ohmic resistance maxima also change with temperature. Consequently, if multilayer or parallel-connected Li-S cells are operated while at different temperatures, significant current and thus SoC imbalances could build up between layers and cells.

Parallel-connected single layer cells.-In Fig. 5a, the terminal voltage of the three parallel single-layer cells held at different temperatures $\left(20,30\right.$, and $\left.40^{\circ} \mathrm{C}\right)$ is shown for fresh cells, after 20 cycles, and after 40 cycles. Figs. 5b-5d illustrate the discharge currents of the three individual cells in parallel for fresh cells and after being cycled. The corresponding charging data is shown in Fig. 6. Fig. 7 compares the charge and discharge curves of the three cells cycled individually at $30^{\circ} \mathrm{C}$, at various points in the cycling procedure, in order to characterize their ageing.

Cells discharged at different temperatures in a parallel connection provide relatively similar current in the upper plateau, Figs. 5b-5d. This is to be expected; in the hotter cell, the smaller resistance is compensated by the decreasing open circuit voltage and by the increase of the ohmic resistance as it discharges. In other words, the increase in ohmic resistance with discharge in the upper plateau, as shown in Fig. 4a, creates a self-balancing effect between parallel cells discharged at different temperatures. However, as soon as the hotter cell reaches the lower plateau, the voltage stops decreasing and the ohmic resistance decreases. As a result, a significant current imbalance emerges between the three cells, with the $40^{\circ} \mathrm{C}$ cell providing more than three times the current drawn from the $20^{\circ} \mathrm{C}$ cell. Towards the end of discharge, the current contributions are reversed: the current provided by the $40^{\circ} \mathrm{C}$ cell drops significantly below those provided by the cells at $20^{\circ} \mathrm{C}$ and $30^{\circ} \mathrm{C}$. This behavior can be explained as follows. Because the $40^{\circ} \mathrm{C}$ cell supports, on average, a higher current, it reaches the voltage-drop toward the end-of-discharge earlier than the colder cells. Due to the parallel connection, the associated drop in its open-circuit voltage and increase in its charge-transfer and transport resistances mean less current is provided by the $40^{\circ} \mathrm{C}$ cell. The colder cells compensate by providing a larger discharge current.

Surprisingly, the parallel connection exhibits a larger discharge capacity after 20 ageing cycles than when fresh, despite the fact that all three cells had been degraded and had less capacities than before cycling, as indicated by Figs. 7a-7b. From Fig. 7b, after 20 ageing cycles the $40^{\circ} \mathrm{C}$ cell suffers most degradation: its resistance increases significantly and its capacity decreases below that of the $20^{\circ} \mathrm{C}$ and $30^{\circ} \mathrm{C}$ cells. The charge throughput to each cell during the first ageing discharge is plotted in Fig. 8a, as calculated from the individual currents in Fig. 5b. Maintaining the cells at different temperatures while 
a)

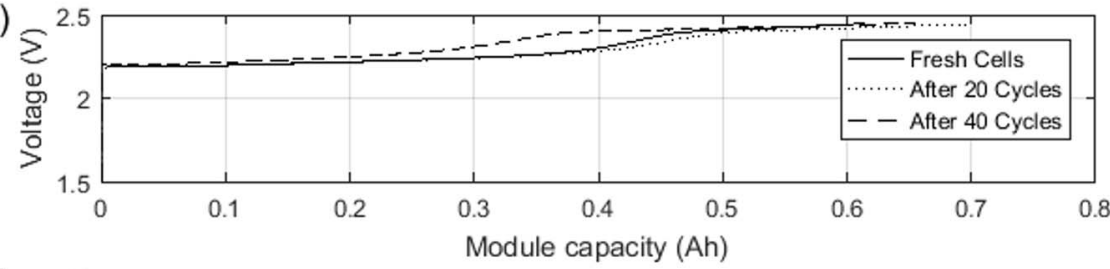

b)

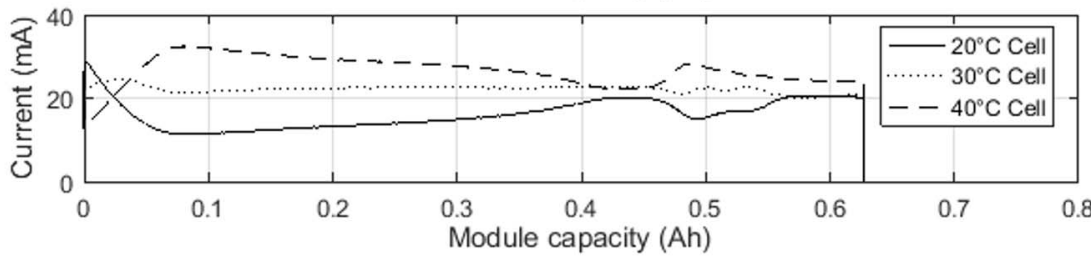

c)

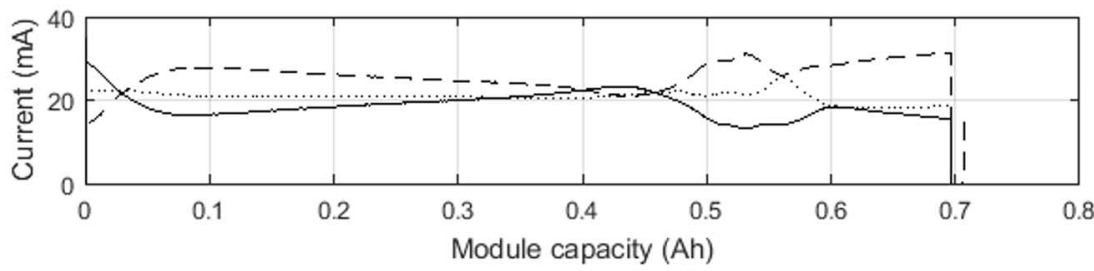

d)

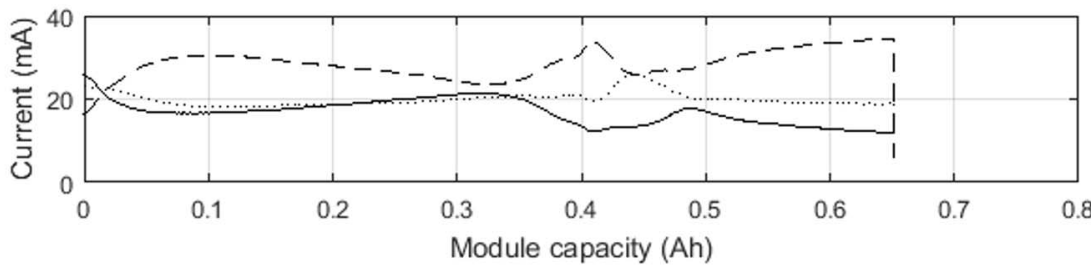

a)

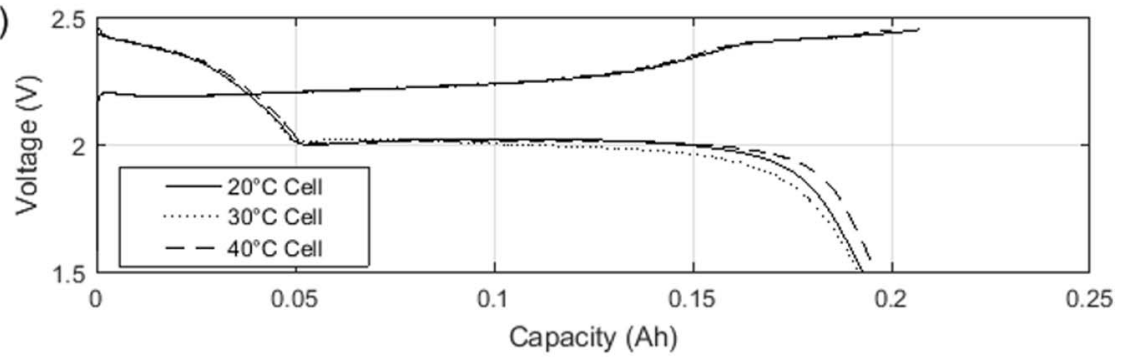

b)

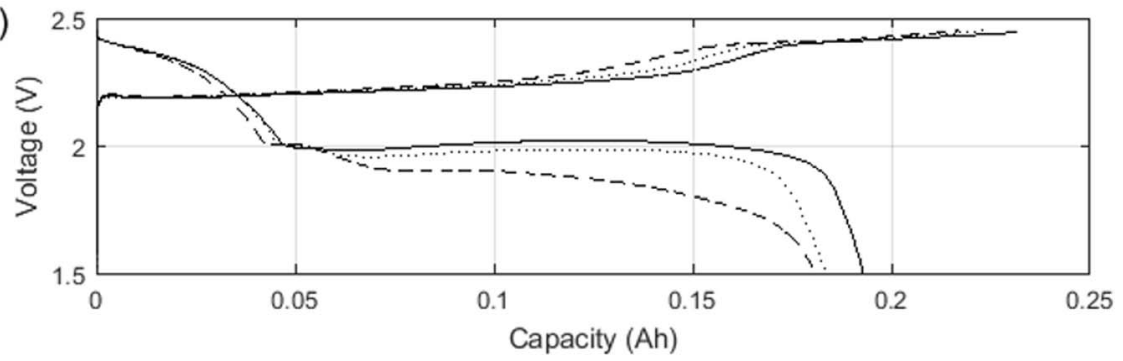

c)

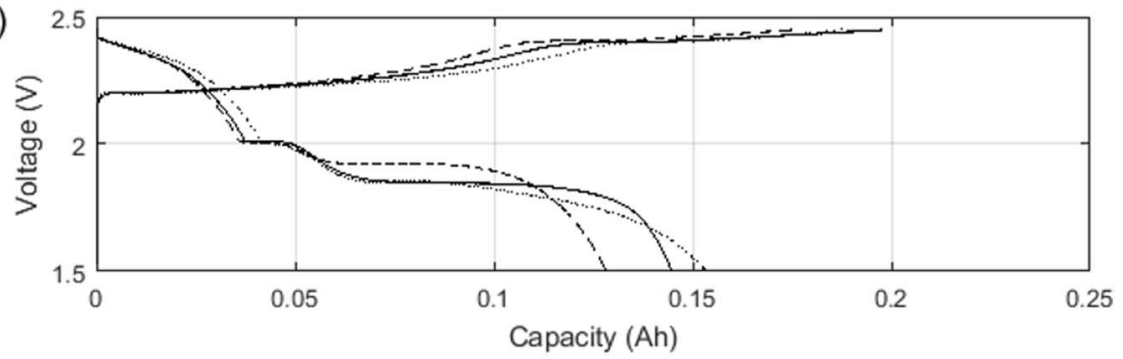

Figure 6. Charge voltage of three parallel-connected 0.211 Ah single layer cells (a) and individual current through each of them during cycle 1, (b) after 20 ageing cycles, and (c) after 40 ageing cycles, for an $0.1 \mathrm{C}$ charge current with $1.5 / 2.45 \mathrm{~V}$ voltage cutoff.
Figure 7. Voltage during $0.2 \mathrm{C} / 0.1 \mathrm{C}$ discharge/charge at $30^{\circ} \mathrm{C}$ of previously parallel-connected single-layer cells held at different temperatures, at various points in the cycling procedure: (a) Fresh cells, (b) after 20 ageing cycles, (c) after 40 ageing cycles. 
(a)

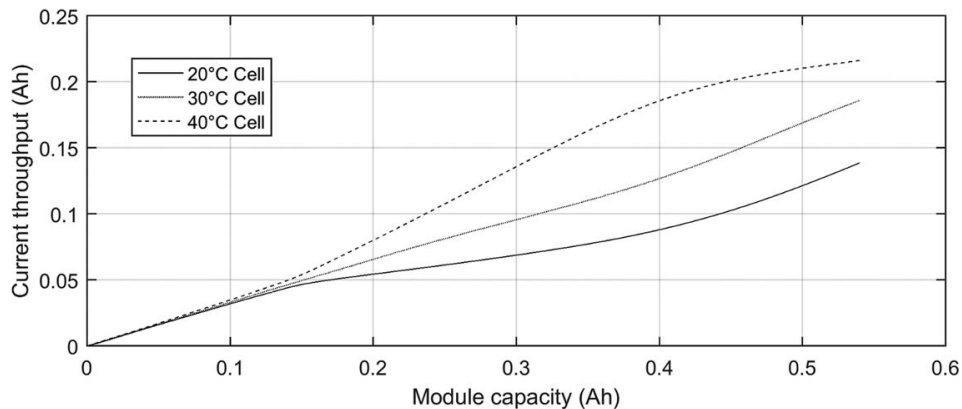

(b)

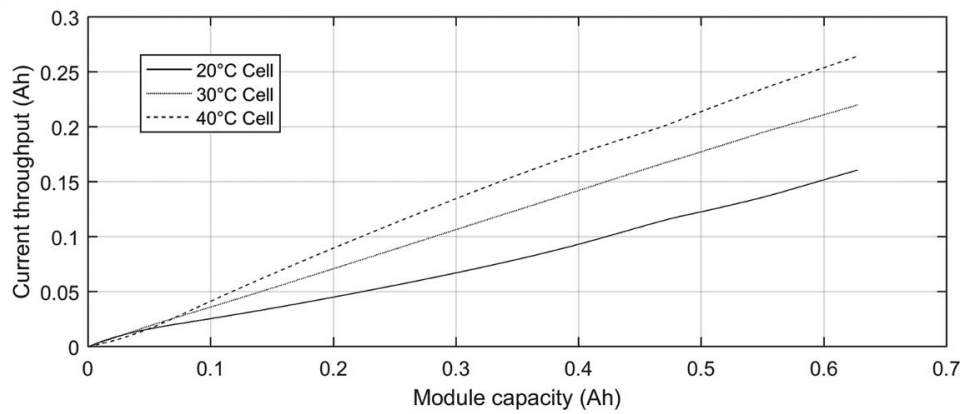

Figure 8. Charge throughput through the three parallel connected single layer cells, during cycle 1 discharge, corresponding to Figure $5 \mathrm{~b}$, and cycle 1 charge, corresponding to Figure $6 \mathrm{~b}$. For both discharge and charge the hotter cell is used considerably more than the other two. connected in parallel leads to a difference in their usable capacity, with the hotter cell discharged most. This remains the case for all further ageing cycles, because the net current through the hotter cell is always larger.

After the characterization cycle, when all three cells are reconnected in parallel, this most degraded cell is again the one maintained at the highest temperature. The difference in their resistance caused by the different temperature is partially compensated by the change in their resistance induced by degradation. As a result, the parallel-connected cells held at different temperatures behave more homogenously after some degradation, as apparent from the smaller spread in current, when comparing Figs. $5 \mathrm{~b}$ and $5 \mathrm{c}$. The same can be concluded by comparing the percentage discharge capacity provided by each cell before and after cycling, shown in Table I. Due to the more homogeneous behavior, the maximum current at the end of the discharge for the $40^{\circ} \mathrm{C}$ cell was lower after 20 ageing cycles, resulting in a smaller voltage drop and therefore an increase in the useable capacity of the parallel connection within the fixed voltage window. After a further 20 ageing cycles, despite the cells exhibiting more homogenous behavior than when fresh, the discharge capacity of the parallel connection is smaller, because all three cells are significantly degraded, Figure 5d.

Due to current inhomogeneity, the parallel cells are at different SoCs at the end of discharge. The cells may also continue to balance each other and currents flow between them when the external load is removed if they are left in this state, although the extent of this effect is not studied here. Consequently, the cells behave differently at the beginning of charge, with the colder cell accepting more current than the hotter cell, as shown in Fig. 6b. The current contributions soon invert, with the $40^{\circ} \mathrm{C}$ cell accepting more current than the $20^{\circ} \mathrm{C}$ cell. Because the cells' ohmic resistances increase during charge throughout the lower plateau, as shown in Fig. 4b, the current imbalance among the cells gradually reduces, similar to what happens in the high plateau

Table I. Percentage of discharge capacity provided by each cell.

\begin{tabular}{lccc} 
& $20^{\circ} \mathrm{C}$ Cell & $30^{\circ} \mathrm{C}$ Cell & $40^{\circ} \mathrm{C}$ Cell \\
\hline Fresh Cells & 25.65 & 34.37 & 39.98 \\
After 20 Cycles & 31.74 & 33.01 & 35.25 \\
After 40 Cycles & 31.59 & 31.69 & 36.72
\end{tabular}

during discharge. The balancing effect disappears once the cells go past the transition point between plateaus, such that the cells' currents diverge again.

The charge throughput for the three cells during the first charging cycle is calculated from the currents in Fig. 6b and illustrated in Fig 8b. Similar to the case for discharge, the hotter cell sees a higher charge throughput. This, together with the fact that this cell reaches the higher plateau sooner, strongly indicates that a considerable fraction of the accepted charge is lost through shuttle. Moreover, shuttle current has been shown to be considerably larger for larger temperatures. ${ }^{12}$ Therefore, the significantly larger degradation of the $40^{\circ} \mathrm{C}$ cell compared to the other two cells is probably caused by the prolonged time it spends within an intensified shuttle region. Another possible contribution to the faster capacity fade of the $40^{\circ} \mathrm{C}$ cell is the incomplete dissolution of precipitated $\mathrm{Li}_{2} \mathrm{~S},{ }^{23}$ as it is charged with a higher average current.

The degradation of the $40^{\circ} \mathrm{C}$ cell temporarily led to a more homogeneous current distribution after 20 cycles, but the continuing cell degradation eventually led to a lower capacity of the parallel connection after 40 cycles. Moreover, as the cells aged, the shuttle became more dominant in the higher plateau, as evident from the larger apparent capacity of the higher plateau after 20 and 40 cycles in Fig. 6. As a consequence coulomb, counting and voltage limits are not sufficient for the safe and efficient operation of Li-S cells, and more advanced model-based control is required instead, in order to accurately predict the state of each layer and/or cell within a real Li-S battery pack.

It should be noted that extracting SoC information from the data presented in Fig. 8b is particularly difficult - the starting conditions for the three cells are different, and depend on all previous cycles, and the percentage of lost charge though shuttle is unknown and different from one cell to another.

Multilayer cell behavior.-The temperature differences artificially induced in this paper correspond to thermal gradients of $20^{\circ} \mathrm{C}$, which are highly unlikely to occur in a realistic cell or pack configuration in a real world application. Such large thermal gradients were induced here so that the response was large enough to study and understand. Therefore, to prove that these effects are still present and to test how significant they might be in a real multi-layer pouch cell, additional complimentary experiments were conducted. The multilayer cell described in the Experimental section above was tested to determine if the middle layers of a multi-layer pouch cell provide more current than the outer layers. 
a)

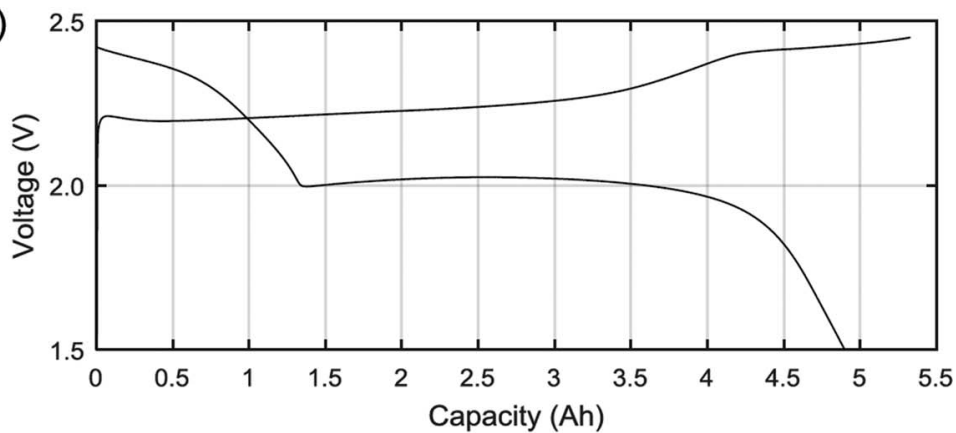

b)

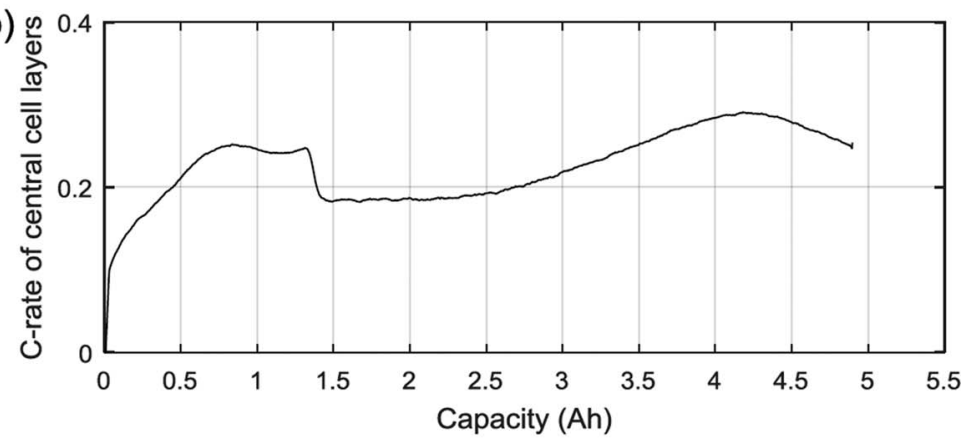

c)

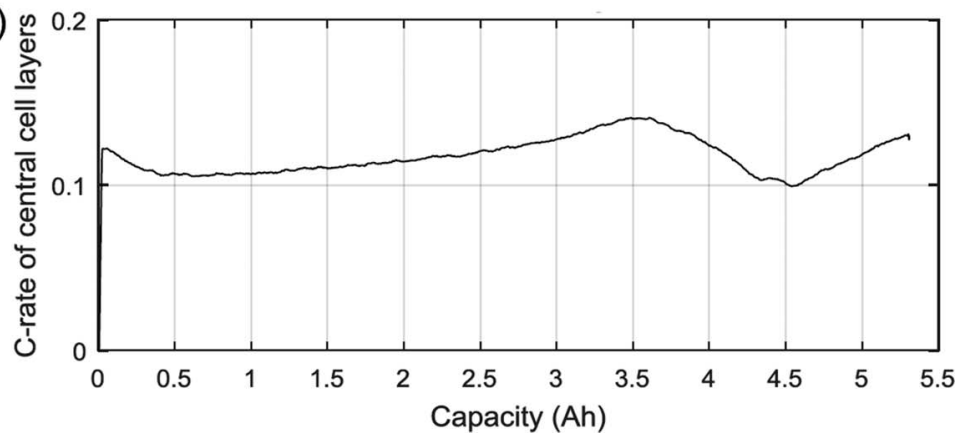

Figure 9. (a) The voltage and (b,c) current loads of the middle three layers of a $6.4 \mathrm{Ah}$ multilayer cell, during the $0.1 \mathrm{C} / 0.2 \mathrm{C}$ charge/discharge with a $1.5 \mathrm{~V} / 2.45 \mathrm{~V}$ voltage cutoff. Data was collected from a bespoke multi-tab cell as illustrated in Figure 3. Despite the absence of significant thermal gradients, the current distribution between the layers of the cell is significantly unequal and evolves during operation.
The current provided by the middle three layers of a 28 layer cell is shown in Fig. 9 during the $5^{\text {th }}$ charge and discharge. The large deviation from the $0.2 \mathrm{C} / 0.1 \mathrm{C}$ discharge/charge load demonstrates that the current load is not equally distributed across the parallel layers of the cell. Due to the relatively small charge and discharge rates, it is unlikely that the significant current inhomogeneity is caused solely by the thermal gradient. In fact, the current evolution does not resemble that of either the hotter or the colder parallel connected single-layer cell shown in Figs. 5-6. The difference in the capacity, resistance, and the SoC of each cell layer could all contribute to the observed inhomogeneity. Therefore, based on the effects of current inhomogeneities observed in parallel-connected single layer Li-S cells, it is expected that the layers also degrade at different rates in a multilayer pouch cell, decreasing the cycle life of the whole cell, which is determined by its worst performing layer. Therefore this effect cannot be ignored when designing battery packs using Li-S cells; and combined with previous work, ${ }^{14}$ wherever possible, we recommend tab cooling rather than surface cooling for Li-S cells. If tab cooling is not effective due to the cell design, then the cells should be redesigned to enable tab cooling.

\section{Conclusions}

It has been shown that for Li-S batteries the charge and discharge voltage curves, as well as the resistance of the cells, are strongly dependent on temperature. By using pouch cells consisting of a single anode and cathode pair and a Peltier-based thermal control system, it is possible to eliminate the effect of a thermal gradient on the behavior of individual cells during charge/discharge.
Voltage and current measurements on parallel-connected singlelayer cells held at different temperatures indicate significant current inhomogeneity among the cells. During discharge, the inhomogeneity is small in the upper voltage plateau, but becomes large in the lower plateau due to positive feedback as the first layer to reach the transition experiences a decreasing cell resistance with SoC. During charge, the cells start at different SoCs, as left by the previous discharge, and the current inhomogeneity decreases in the lower plateau and increases in the upper plateau; again because of the effect of the mountain-shape resistance increasing resistance until the transition and then decreasing resistance as Li-S cells approach $100 \%$ SoC. Consequently, the hotter Li-S single layer cell accepts, on average during charge, more current than the other two cells in parallel. Due to its lower resistance and fixed voltage cutoff, it is forced into increasingly shuttle dominated behavior toward the end of charge. This causes the hotter cell to degrade faster than colder cells, despite all being cycled in parallel. The different rates of degradation initially cause the parallel single-layer cells to behave more homogeneously under thermal gradient, but continued cycling with thermal and current inhomogeneity caused severe degradation in all cells, and thus also in the capacity of the parallel system.

Current measurements on a bespoke multilayer pouch cell with separate tabs for the central three layers suggests significant current inhomogeneity could arise in practical Li-S pouch cells, even in the absence of artificially induced thermal gradients. The current inhomogeneity between parallel Li-S cells must be accounted for when modelling the performance and degradation of multilayer Li-S cells and Li-S battery packs, in order for model predictions to be valid. Understanding the effect of thermal and current homogeneity on cell 
degradation is also crucial for the design of thermal management system for Li-S batteries, and wherever possible we would recommend tab cooling rather than surface cooling for Li-S cells, and if tab cooling is not effective due to the cell design then the cells should be redesigned to enable tab cooling.

\section{Acknowledgments}

The authors thank Innovate UK and the Engineering and Physical Sciences Research Council in the UK for funding this work under the Revolutionary Electric Vehicle Battery (REVB) project 101573 and EP/L505298/1.

\section{ORCID}

M. Marinescu (D) https://orcid.org/0000-0003-1641-3371

G. J. Offer (D) https://orcid.org/0000-0003-1324-8366

\section{References}

1. P. G. Bruce, S. A. Freunberger, L. J. Hardwick, and J.-M. Tarascon, Nat. Mater, 11, 19 (2012).

2. D. P. Finegan et al., Nat. Commun., 6, 6924 (2015).

3. X. Ji and L. F. Nazar, J. Mater. Chem., 20, 9821 (2010).
4. I. A. Hunt, Y. Patel, M. Szczygielski, L. Kabacik, and G. J. Offer, J. Energy Storage, 2, 25 (2015)

5. M. Wild et al., Energy Environ. Sci., 8, 3477 (2015)

6. S. S. Zhang, J. Power Sources, 231, 153 (2013).

7. H. Kim, J. T. Lee, and G. Yushin, J. Power Sources, 226, 256 (2013).

8. X. Li et al., Nano Lett, 16, 3545 (2016).

9. M. Ghaznavi and P. Chen, J. Power Sources, 257, 402 (2014).

10. H.-S. Ryu et al., J. Power Sources, 163, 201 (2006).

11. M. Barghamadi, A. Kapoor, and C. Wen, J. Electrochem. Soc., 160, A1256 (2013).

12. Y. V. Mikhaylik and J. R. Akridge, J. Electrochem. Soc., 151, A1969 (2004).

13. J. Seo, C.S. Kim, J. Prakash, and K. Zaghib, Electrochem. commun., 44, 42 (2014).

14. I. A. Hunt, Y. Zhao, Y. Patel, and J. Offer, J. Electrochem. Soc., 163, A1846 (2016).

15. R. R. Richardson, P. T. Ireland, and D. A. Howey, J. Power Sources, 265, 254 (2014).

16. X. Yang et al., Adv. Funct. Mater, 26, 8427 (2016)

17. X. Yang et al., Nano Energy, 39, 418 (2017).

18. M. R. Busche et al., J. Power Sources, 259, 289 (2014).

19. J. R. Akridge, Y. V. Mikhaylik, and N. White, Solid State Ionics, 175, 243 (2004).

20. T. Zhang, M. Marinescu, S. Walus, P. Kovacik, and G. J. Offer, J. Electrochem. Soc., 165, A6001 (2018)

21. T. Zhang, M. Marinescu, L. O'Neill, M. Wild, and G. Offer, Phys. Chem. Chem. Phys., 17, 22581 (2015).

22. V. S. Kolosnitsyn, E. V. Kuzmina, E. V. Karaseva, and S. E. Mochalov, J. Power Sources, 196, 1478 (2011).

23. M. Marinescu, T. Zhang, and G. J. Offer, Phys. Chem. Chem. Phys, 18, 584 (2016). 